\title{
MicroRNAs Indirectly Regulate Other MicroRNAs in Ovarian Cancer Cells
}

\author{
Shubin W. Shahab ${ }^{1,2}$, Lilya V. Matyunina $a^{1,2,3}$, Vinay K. Mittal ${ }^{1,2}$, \\ Lijuan Wang $^{1,2}$, Christopher G. Hill ${ }^{1,2}$, L. DeEtte Walker ${ }^{1,2,3}$ \\ and John F. McDonald ${ }^{1,2,3^{*}}$ \\ 'School of Biology, Georgia Institute of Technology, Atlanta, GA, USA. \\ ${ }^{2}$ Parker H. Petit Institute for Bioengineering and Bioscience, \\ Georgia Institute of Technology, Atlanta, GA, USA. \\ ${ }^{3}$ Ovarian Cancer Institute, Atlanta, GA, USA.
}

Research Article

Received 28 ${ }^{\text {th }}$ August 2011

Accepted $25^{\text {th }}$ January 2012

Online Ready $21^{\text {st }}$ February 2012

\section{ABSTRACT}

MicroRNAs (miRNAs) are a class of regulatory RNAs that control the expression of genes critical to cell function. Ectopic expression of miRNAs has been shown to result in genome-wide changes in patterns of gene expression. While the reasons for these global alterations in gene expression patterns have been attributed to the ability of miRNAs to target multiple genes, and/or to induce indirect effects downstream of target genes, the molecular basis of indirect effects of miRNA regulation remains poorly understood. In this study, we demonstrate the potential of miRNAs to regulate other miRNAs. Using miRNA microarray analysis, we show that over 70 different miRNAs are differentially expressed ( $\geq 1.4$ fold, FDR $\leq 5 \%)$ in human ovarian cancer cells after transfection with a single miRNA (miR-7). We present evidence that a major component of miR-7 induced changes in levels of miRNAs is the indirect consequence of miR-7 mediated alterations in levels of protein coding genes (e.g., transcription and splicing factors) that exert trans-regulatory control on miRNAs.

Keywords: miRNA; ovarian cancer; systems biology; miR-7; NF-кB. 


\section{INTRODUCTION}

MicroRNAs (miRNAs) are a conserved class of small RNAs that can regulate gene expression by altering the translation and/or mRNA stability of target genes (Bartel, 2004; Fabian et al., 2010). Since miRNAs are differentially expressed in cancer and other diseases (Calin and Croce, 2006; Esquela-Kerscher and Slack, 2006; Farazi et al., 2011; Lee and Dutta, 2009; Visone and Croce, 2009; Wiemer, 2007), they may have clinical potential as diagnostic, prognostic and/or therapeutic agents (Garofalo and Croce, 2011; Wahid et al 2010). However, the molecular causes and consequences of perturbations in levels of miRNAs in cancer cells can be varied and complex (Baek et al., 2008; Lim et al., 2005; Selbach et al., 2008; Shahab et al., 2011). Thus, the potential clinical impact that miRNAs will ultimately have in cancer medicine rests heavily upon our ability to understand the basis of this complexity. We report here that perturbations in levels of a single miRNA can not only induce significant changes in levels of mRNAs, but also significantly modulate levels of other miRNAs. We discuss the potential significance of miRNA-miRNA regulation in cancer cells and present evidence from global gene expression and protein analyses for molecular mechanisms that may contribute to the process.

\section{MATERIALS AND METHODS}

\subsection{Cell Culture and miRNA/siRNA Transfections}

Cell culture conditions and miRNA/siRNA transfection procedures were carried out as described previously (Dickerson et al., 2010). Briefly, approximately $12 \mathrm{~h}$ before transfection, cells (duplicates or triplicates per transfection, $1.5 \times 10^{5}$ per well) were seeded on six-well plates in growth medium RPMI 1640 (Mediatech, Manassas, VA) supplemented with $10 \%$ $\mathrm{v} / \mathrm{v}$ heat-inactivated fetal calf serum (Invitrogen, Carlsbad, CA), $2 \mathrm{mM}$ L-glutamine (Mediatech), $10 \mathrm{mM}$ HEPES buffer (Mediatech), penicillin $(100 \mathrm{U} / \mathrm{ml})$, and streptomycin $(100 \mu \mathrm{g} / \mathrm{mL})$ and allowed to adhere overnight at $37^{\circ} \mathrm{C}$ in a $5 \% \mathrm{CO}_{2}$ atmosphere. The following day after washing the wells with PBS and replacing the growth medium with OptiMEM (Invitrogen), cells were transfected with the miRNA or siRNA [hsa-miR-7 miRIDIAN mimic (cat \# C-300546-07), miRIDIAN miRNA mimic negative control \#1 (miR-NC; cat \# CN001000-01, is a C.elegans miRNA, cel-miR-67, with minimal sequence identity in humans), ON-TARGET plussiRNARELA (cat \# J-003533-06) or ON-TARGET plus non-targeting siRNA (siNC, cat \# D-001700-01) (Thermo Fisher Scientific, Lafayette, CO)] using Lipofectamine 2000 transfection agent (Invitrogen, Carlsbad, CA) according to the manufacturer's instructions at a final concentration of $25 \mathrm{nM}$. All transfections were carried out in triplicate. The cells were incubated with the reduced serum transfection medium (OptiMEM, Invitrogen) for 4 hours, washed and then allowed to grow in growth medium (RPMI 1640) for 44 hours before collecting RNA or protein. Transfection efficiency was estimated from the relative knock-down of EGFR/RELA, based on recommendations by the reagent manufacturer (Thermo Fisher Scientific).

\subsection{Quantitative (Real-time) PCR}

For miRNA qPCR, total RNA was extracted from HEY cells using the mirVana miRNA isolation kit according to the manufacturer's instructions (Applied Biosystems, Foster City, $\mathrm{CA}$ ). The RNA (10 $\mathrm{ng}$ ) was then converted to amplified cDNA for qPCR by using the TaqMan miRNA Reverse Transcription Kit and miRNA specific primers (Applied Biosystems) following manufacturer's protocols. TaqMan miRNA assays (Applied Biosystems) were 
conducted following manufacturer's protocol ( $20 \mu \mathrm{l}$ total volume) forhsa-miR-198, hsa-miR628-3p, hsa-miR-149, hsa-miR-10b, hsa-let-7f, hsa-miR-503, hsa-miR-218, hsa-miR224, and for RNU6B. Specificity of TaqMan miRNA assays is supported by publications from the manufacturer (Chen et al., 2007; Liang et al., 2007; Tavazoie et al., 2008) as well as subsequent literature from independent groups (Huang et al., 2011; Shibata et al., 2011; Wilson et al., 2011).

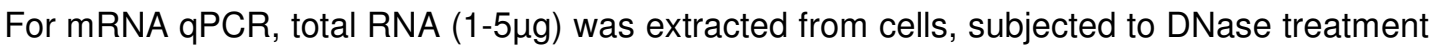
(Qiagen, Valencia, CA) and converted to cDNA using the Superscript III First Strand synthesis system (Invitrogen). cDNA was then purified using the Qiagen PCR purification kit following manufacturer's instructions. TaqMan gene expression assays were conducted following manufacturer's protocol for MGC16121, FSTL1, GAPDH, RELA, and SRSF1. qPCR experiments were also carried out for the EGFR and GAPDH genes using $\mathrm{iQ} \mathrm{SYBR}$ Green Supermix (Bio-Rad, Hercules, CA). The sequence specific primers used for EGFR are described in (Micallef et al., 2009) and GAPDH primers are described in (Koppelstaetter et al., 2005). All reactions were optimized with non-template controls, and -RT (no reverse transcriptase) controls prior to the experiment. Specificity of TaqMan gene expression assays is supported by thousands of independent citations (e.g., (Bergmann et al., 2011; Colazzo et al., 2011; Hevir et al., 2011; Lossos et al., 2004).

All qPCR experiments were carried out using at least 3 technical replicates and 2-3 independent biological replicates on the CFX96 Real Time PCR detection system (Bio-Rad). For each targetexpression values were normalized to an endogenous control (RNU6B/GAPDH). RNU6B is a previously established control for human ovarian samples (http://www.ambion.com/techlib/tn/151/3.html;

http://www3.appliedbiosystems.com/cms/groups/mcb_marketing/documents/generaldocu

ments/cms_044972.pdf). GAPDH was chosen as endogenous control because it displays minimal change between HEY cells transfected with miR-NC and miR-7 (Shahab et al., 2011). Relative fold change of target RNA level between transfection groups was determined by the $\Delta \Delta$ Ctmethod. Statistical significance was determined using the pair-wise fixed reallocation randomization test in the Relative Expression Software Tool (REST 2008)(Pfaffl et al., 2002).

\subsection{Immunoblotting}

Immunoblots for EGFR, RELA/NFkB, SRSF1 and $\beta$-Actin were performed as described previously (Blackburn et al., 2009) with the following modifications. The blots were incubated for 2 hours with goat anti-rabbit IgG (1:2000) linked to Fluorescein (FITC; Southern Biotech, Birmingham, $A L)$, or with donkey anti-mouse IgG (1:5000) linked to Phycoerythrin (PE; Southern Biotech, Birmingham, AL) secondary antibodies at room temperature. Primary antiEGFR and anti-Actin antibodies were described in (Blackburn et al., 2009). Primary antiRELA antibody was purchased from Santa Cruz Biotechnology (Santa Cruz, CA; sc-372) and primary anti-SRSF1 antibody was purchased from Millipore (Billerica, MA; 06-1363). Bands were visualized using a Typhoon 9400 Imager (GE Healthcare, Piscataway, NJ) followed by densitometric analysis using the ImageQuant TL Software (GE Healthcare). Statistical significance of difference between average levels from at least three replicates quantified by densitometry was determined using a 2-tailed unpaired Student's t-test. 


\subsection{RNA Isolation for miRNA Microarray}

Low molecular weight RNA was isolated from HEY cells (two replicates per treatment using the mirVana miRNA isolation kit according to the manufacturer's instructions (Applied Biosystems). The quantity and size of miRNA was verified using an Agilent 2100 Bioanalyzer (Agilent Technologies, Palo Alto, CA). MiRNAs $(200 \mu \mathrm{g} / \mathrm{sample})$ were labeled with the GenisphereFlashTag HSR Biotin RNA labeling kit (Genisphere, Hatfield, PA) followed by hybridization with GeneChip miRNA Arraychips (Affymetrix, Santa Clara, CA) according to the manufacturer's instructions. The chips were washed and then scanned with a GeneChip Scanner 3000 (Affymetrix). Raw data in the form of CEL files were produced by AffymetrixGeneChip Operating System (GCOS) software.

\subsection{RNA Isolation for Whole Genome Microarray}

Total RNA for whole genome microarray was isolated using the RNeasy Mini RNA isolation kit (Qiagen) followed by DNAse treatment according to manufacturer's instructions. The integrity of the RNA was verified using an Agilent 2100 Bioanalyzer (RIN 8.0-9.8); Agilent Technologies). mRNAs were converted to double stranded cDNA and amplified using the Applause 3'-Amp System (NuGen, San Carlos, CA). This cDNA was then biotin labeled and fragmented by using the Encode Biotin Module (NuGen). The labeled cDNA was hybridized to Affymetrix HG-U133 Plus 2.0 oligonucleotide arrays and analyzed with a GeneChip Scanner 3000 (Affymetrix). Raw data in the form of CEL files were produced by AffymetrixGeneChip Operating System (GCOS) software.

\subsection{Microarray Data Analysis}

miRNA microarray data were analyzed using miRNA QC Tool software (Affymetrix). Normalization was performed using the default workflow of the software. The $\log _{2}$ transformed expression values were then normalized across samples by Z-score calculations using Spotfire DecisionSite for Microarray Analysis (DSMA). Probesets with "Absent" call in all groups were removed from analysis. Probeset intensities were filtered with DSMA to include only those probesets with a fold change $\geq 1.4$. Differentially expressed probesets were identified using the SAM algorithm at a threshold $5 \%$ false discovery rate (FDR) correction. Sequence of each differentially expressed miRNA probeset was then used to find the corresponding human miRNA using the BLASTN algorithm (http://www.mirbase.org/search.shtml) with E-value cut-off of 10. In each case, only the human miRNA with the highest score and lowest E-value was considered. If two miRNAs had the same high score, then both were included. Sequences with $1<\mathrm{E}$-value $<10$ were included in the table (see results) but were excluded from further analysis because of their "poor homology". Sequences with E-value $>10$ for human miRNAs are ignored and marked with "N/A" under the 'homolog' columns in results. Genomic locations of miRNA paralogs were obtained from the miRBase registry as well. In the miR-7 transfection experiment these probesets correspond to 71 unique mature human miRNAs (excluding miR-7), 3 sequences with very poor (BLASTN E-value >1) homology to human miRNAs, 2 snoRNAs, 1 vault RNA, 1 tRNA, 1 KIAA1407 RNA fragment, and some sequences that we could not map to the human genome (E-value $>10)$.

mRNA microarray data were analyzed using Expression Console (Affymetrix) and R (www.rproject.org). Normalization was performed using MAS 5.0, and PLIER (Expression Console) and GCRMA algorithm (R). The $\log _{2}$ transformed expression values from MAS5.0 were 
analyzed for Affymetrix "Present/Absent" calls using Spotfire DecisionSite for Microarray Analysis (DSMA). Probe sets with "Absent" call in all groups were removed from analysis. Average probe set intensities for each group were calculated based on the $\log _{2}$ transformed values from PLIER and then filtered with DSMA to include only those probe sets with a fold change $\geq 1$. 3 . The t-test $p$-value for each probe set was calculated from the $\log _{2}$ transformed values after GCRMA normalization using DSMA. Finally, differentially expressed probesets were identified using a threshold fold change $\geq 1.3$, t-test $p$-value $<0.05$ and "Present/Marginal" call in at least one sample. These three different filtering approaches were used based on recommendations from a recent publication (Mieczkowski et al., 2010) and the combination of all three was used to achieve the most stringent filtering.

Raw data from a previous miR-7 transfection paper (Webster et al., 2009) were downloaded from GEO (Gene Expression Omnibus; http://www.ncbi.nlm.nih.gov/geo/) and processed in a similar fashion. All microarray data are MIAME compliant and have been submitted to GEO under the accession nos. GSE29129 (miRNA) and GSE27431 (mRNA).

\subsection{Identification of Transcription Factors}

Transcription factors were identified by searching for the Gene Ontology (GO) biological process annotation "regulation of transcription" for every probeset differentially expressed after miR-7 transfection.

\section{8 miRNA Target Download}

The miRNA target prediction file based on miRanda-mirSVR was downloaded from www.microrna.org (August 2010 release) (Betel et al., 2008;Enright et al., 2003; John et al., 2004). Information about the prediction algorithm, parameter settings and raw data source is available at the above link.

\subsection{Identification of Putative NF-kBRegulated miRNAs}

The coordinates of the putative NF-KB binding sites identified by ChIP-seq in the ENCODE project (Birney et al., 2007) were downloaded from the UCSC table browser (http://genome.ucsc.edu) based on the NCBI36/hg18 assembly of the human genome for each miRNA using a window of $\pm 10 \mathrm{~kb}$ around the locus of each pre-miRNA corresponding to the differentially expressed miRNAs following miR-7 transfection.

\subsection{Identification of Putative SRSF1 Regulated miRNAs}

SRSF1 binding sites identified by (Sanford et al., 2009) using genome-wide cross-linked immunoprecipitation and sequencing (CLIP-seq) were downloaded from the authors website (with authors permission; http://sanfordlab.mcdb.ucsc.edu/Sanford_Lab/Datasets.html). For miRNAs embedded in known transcriptional units the SRSF1 sites were searched within the genomic coordinates of the host transcript. For intergenic miRNAs, length of the pri-miRNA was assumed to be $\sim 4 \mathrm{~kb}$ on average with the transcription start site located $\sim 2 \mathrm{~kb}$ upstream and the poly-A signal located $\sim 2 \mathrm{~kb}$ downstream of the pre-miRNA, and thus the SRSF1 sites were searched within $\pm 2 \mathrm{~kb}$ around each pre-miRNA, based on the average lengths of pri-miRNAs described by (Saini et al., 2007). Coordinates of coding transcriptional units and pre-miRNAs were obtained from the UCSC genome browser (http://genome.ucsc.edu) based on the $\mathrm{NCBI} 36 / \mathrm{hg} 18$ assembly of the human genome. 


\subsection{BLAST Alignment of miRNAs}

Sequences of miRNA probesets obtained from the Affymetrix annotation file corresponding to the differentially expressed miRNAs following miR-7 transfection were searched for significant complementarity to miR-7 using NCBI nucleotide BLAST algorithm (http://blast.ncbi.nlm.nih.gov) with the following parameter settings. Under program selection, optimize for "more dissimilar sequences" was selected, and under algorithm parameters, "automatically adjust parameters for short input sequences" was selected.

\section{RESULTS}

\subsection{Ectopic Expression of miR-7 Alters the Levels of Endogenous microRNAs}

To study the effect of changes in levels of a single miRNA on expression levels of other miRNAs in cancer cells, we transfected a well-characterized ovarian cancer cell line (HEY) with a miRNA (miR-7) previously shown to be significantly up-regulated in ovarian and other cancers relative to normal (Chou et al., 2010; Jiang et al., 2010; Kefas et al., 2008; Reddy et al., 2008; Saydam et al., 2010; Shahab et al. 2011; Veerla et al., 2009; Webster et al., 2009; Wyman et al., 2009). Using small RNA sequence datasets generated from a number of ovarian cell lines and tissue samples (Creighton et al. 2011, Gene Expression Omnibus, GSE 16709), we determined that the endogenous level of miR-7 in HEY cells is relatively low and generally representative of miR-7 levels in other OC cell lines and primary OC tumors (Figure S1).

To assess the biological effectiveness of our transfection, we monitored changes in levels of EGFR, a validated target of miR-7 (Kefas et al., 2008; Webster et al., 2009). Since it has previously been demonstrated that EGFR is highly expressed in HEY cells (Dickerson et al., 2010), we expected levels of EGFR mRNA and protein to be significantly reduced after a successful miR-7 transfection. Our results confirm that levels of EGFR mRNA and protein are both significantly reduced after miR-7 transfection (Figure 1) relative to a negative control (miR-NC).

To evaluate the effect of miR-7 transfection on global miRNA expression profiles, we isolated total RNA from cells transfected with either miR-7 or a negative control miRNA (miR-NC) 48h after transfection and monitored changes in levels of miRNA expression by microarray analysis. The results demonstrate that 259 distinct miRNA probesets corresponding to 71 mature miRNAs (excluding miR-7) were significantly altered in expression (31 up-regulated; 40 down-regulated) in the miR-7 transfected cells relative to controls (Figure 2, Table S1).

Eight of the miRNAs displaying significant changes in expression by microarray (hsa-miR198, hsa-miR-628-3p, hsa-miR-149, hsa-miR-10b, hsa-let-7f, hsa-miR-503, hsa-miR-218 and hsa-miR-224) were randomly selected for confirmatory quantitative (real-time) PCR (qPCR) analysis. The results were uniformly consistent with the microarray results (Figure S2 A-H).

\subsection{The Majority of Genes in Which Differentially Expressed miRNAs are Embedded Do Not Display Correlated Changes in Expression}



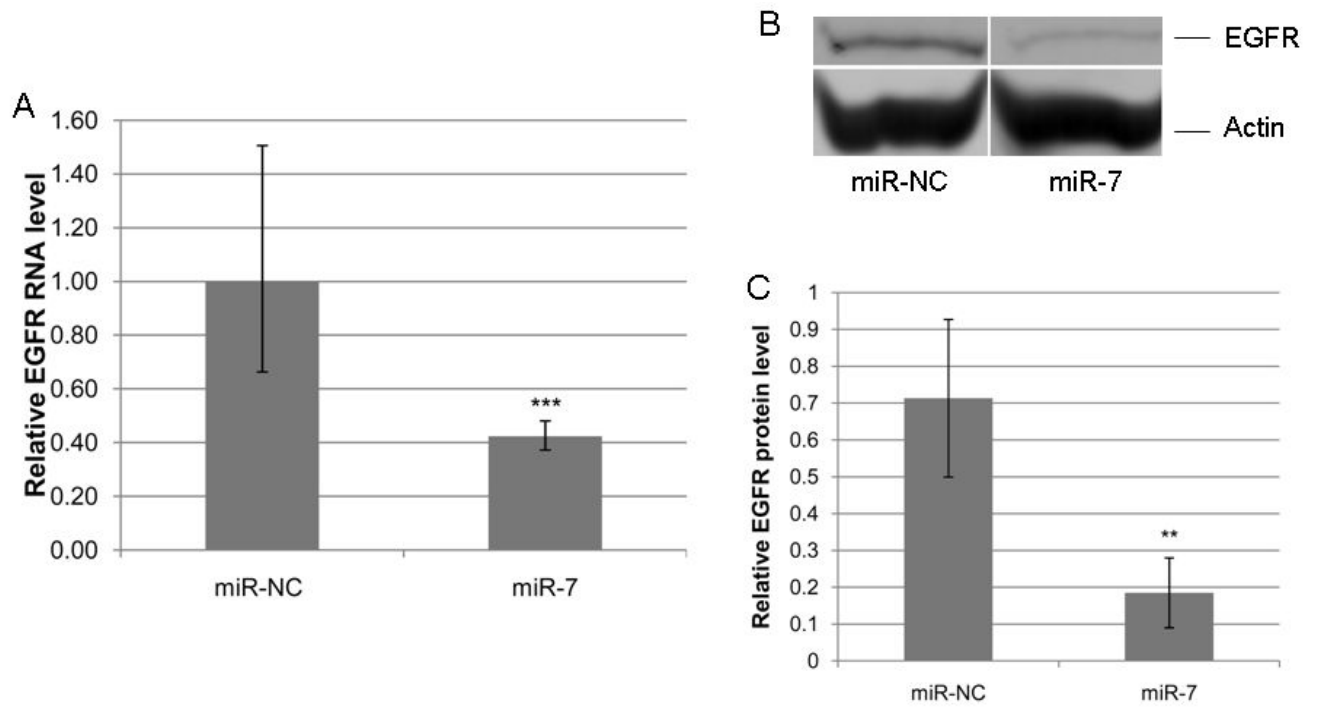

Figure 1. Confirmation of successful transfection of miR-7 into HEY cells.

Successful transfection of miR-7 into HEY cells is confirmed by measuring the level of EGFR RNA by qPCR $(A)$ and protein by Western blot analysis ( $B$ and $C$ ). In both cases EGFR expression is downregulated significantly $\left({ }^{* *} p<0.01 ;{ }^{* * *} p<0.001\right)$ after miR-7 transfection compared to negative control (miR-NC) transfection. Bar graphs in $C$ show the relative intensity of blots in B. EGFR expression is normalized to GAPDH used as endogenous control (A), or $\beta$-Actin used as loading control (C). Error bars reflect standard deviation of the means calculated from 3 independent replicate samples.

Human miRNAs have been mapped to exons and introns of protein coding and non-protein coding genes (i.e., embedded in genes), as well as to intergenic regions of the genome (Kim and Nam, 2006). Some embedded miRNAs have been shown to display changes in expression levels that positively correlate with those of their host genes. Such correlated patterns of expression are consistent with the hypothesis that embedded miRNAs and their host genes are under the same and/or coordinated regulatory controls (Baskerville and Bartel, 2005;Rodriguez et al., 2004). In other cases, changes in patterns of embedded miRNAs do not correlate with those of their host genes (Bargaje et al., 2010) indicating that at least some embedded miRNAs are under independent regulatory control (Fujita and Iba, 2008; Monteys et al., 2010; Ozsolak et al., 2008).

Of the 71 miRNAs differentially expressed after miR-7 transfection, 50 were found to map to annotated genes (55 including paralogs) and 21 (25 including paralogs) map to intergenic regions (Figure 3, Table S2). To determine if changes in miRNA levels are correlated with changes in mRNA levels of the 50 genes in which miRNAs are embedded, we compared changes in miRNA profiles with changes in the mRNA levels of host genes in miR7transfected HEY cells (Table S3).We found that 9 of the 50 host genes were significantlydifferentially expressed (fold change $\geq 1.3, p<0.05$ ) in miR-7 transfected cells relative to controls (Tables 1 and S3, Figure S2 I-J). Eight of these nine genes displayed changes in expression positively correlated with 13 embedded miRNAs while one of the genes displayed a change in expression inversely correlated with a single embedded miRNA (Table 1$)$. The majority of genes (41/50) hosting differentially expressed miRNAs did not display a significant change in expression in miR-7 transfected cells relative to controls (Table S2). 
A

\begin{tabular}{l|l}
\hline \multicolumn{2}{l}{ Down-regulated miRNAs } \\
\hline hsa-miR -424 & hsa-let-7a \\
\hline hsa-miR -424* & hsa-let-7f \\
\hline hsa-miR-503 & hsa-1et-7g \\
hsa-miR-20b & hsa-miR-199a (3p) \\
hsa-miR-7-1* & hsa-miR-199a (5p) \\
hsa-miR-199b-3p & hsa-miR-98 \\
hsa-miR-224 & hsa-miR-17 \\
hsa-miR-29b-1* & hsa-miR-92a \\
hsa-miR-1274a & hsa-miR-92a-1* \\
hsa-miR-494 & hsa-miR-193a-3p \\
hsa-miR-30a* & hsa-miR-126 \\
hsa-miR-218 & hsa-miR-1224-5p \\
hsa-miR-155 & hsa-miR-10b \\
hsa-miR-221* & hsa-miR-15b \\
hsa-miR-584 & hsa-miR-100* \\
hsa-miR-374b & hsa-miR-30d* \\
hsa-miR-198 & hsa-1et-7c \\
hsa-miR-223 & hsa-miR-10a \\
hsa-miR-204 & hsa-miR-137 \\
hsa-miR-139-5p & hsa-miR-30e* \\
\hline
\end{tabular}

\begin{tabular}{ll}
\hline \multicolumn{2}{l}{ Up-regulated miRNAs } \\
\hline hsa-1et-71* & hsa-miR-421 \\
hsa-miR-1180 & hsa-miR-425* \\
hsa-miR-1246 & hsa-miR-542-5p \\
hsa-miR-1303 & hsa-miR-550a-1 \\
hsa-miR-140-5p & hsa-miR-551b \\
hsa-miR-142-5p & hsa-miR-590-5p \\
hsa-miR-146a & hsa-miR-628-3p \\
hsa-miR-149 & hsa-miR-652 \\
hsa-miR-181a-2* & hsa-miR-877 \\
hsa-miR-181b-2 & hsa-miR-93* \\
hsa-miR-301b & hsa-miR-99b* \\
hsa-miR-181c & hsa-miR-330-3p \\
hsa-miR-181d & hsa-miR-337-5p \\
hsa-miR-200b & hsa-miR-338-3p \\
hsa-miR-210 & hsa-miR-33a \\
hsa-miR-26b* & \\
\hline
\end{tabular}

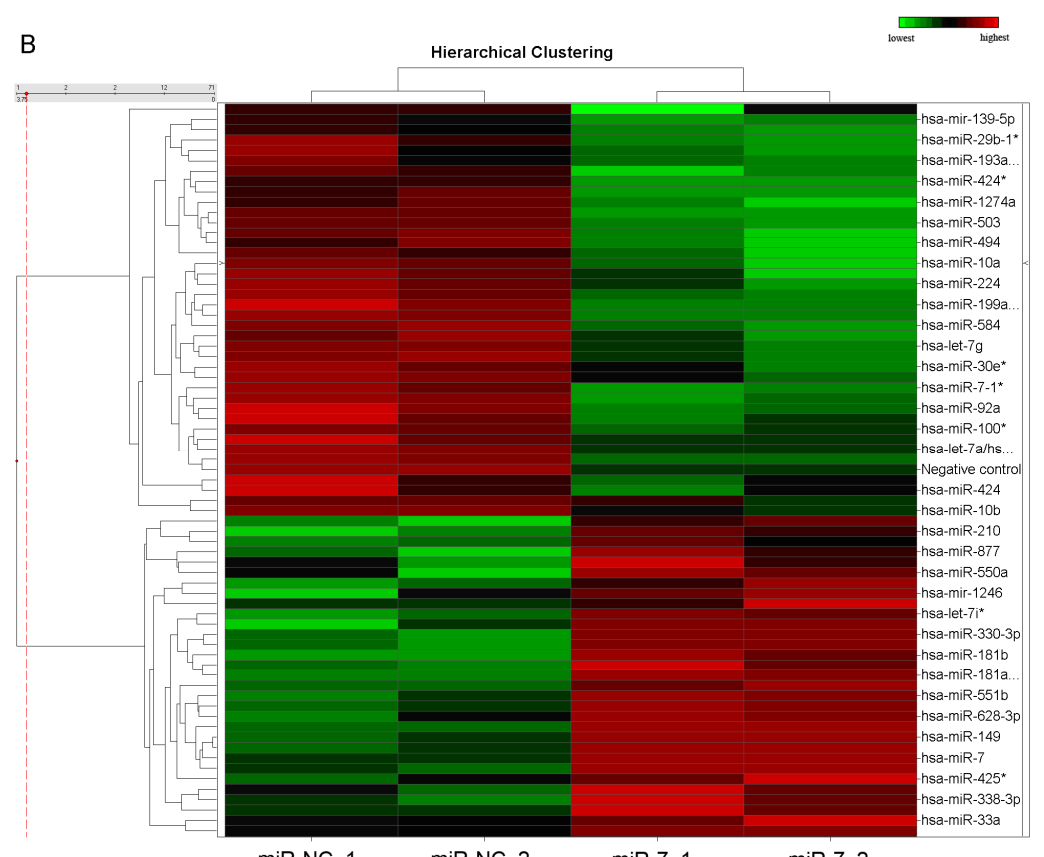

miR-NC

miR-NC_2

miR-7_1

miR-7_2

Figure 2. MicroRNAs differentially expressed after miR-7 transfection in HEY cells. A. List of differentially expressed (fold change $\geq 1.4$ and $F D R \leq 5 \%$ ) miRNAs following transfection of hsa-miR-7 into HEY cells compared to miR-NC transfected cells. There were 40 different human miRNAs significantly down-regulated and 31 miRNAs significantly up-regulated (excluding miR-7) after miR-7 transfection. MicroRNAs mapping to the same transcriptional unit are shaded with the same color (See Table S2 for additional details) B. Hierarchical clustering of significantly differentially expressed miRNAs between cells transfected with either miR-7 or miR-NC. The two negative control samples are on the left and the two miR-7 transfected samples are on the right. Selected IDs of the human miRNAs homologous to thesequences specified by the probesets are shown on the right side of the heat map. [Note some of the miRNA names have an asterisk (*) indicating miRNA star strand]. 


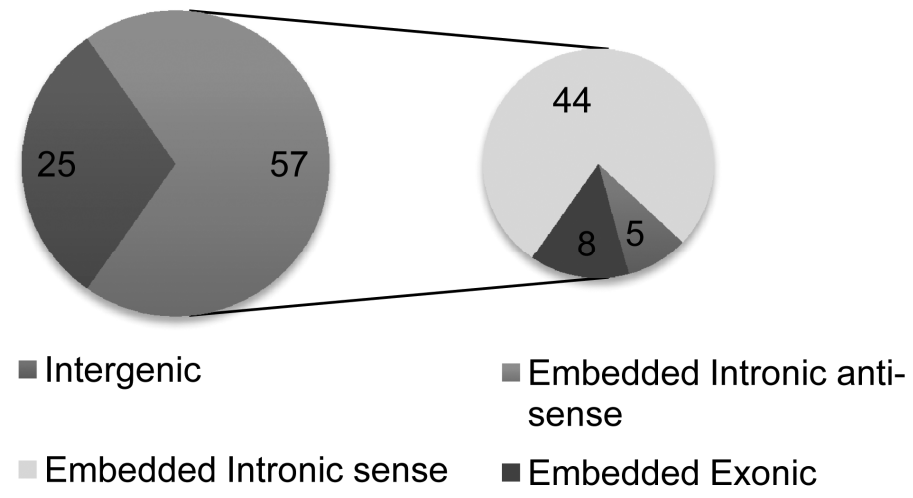

Figure 3. Genomic location of differentially expressed miRNAs between miR-7 transfected and negative control transfected HEY cells

Table 1. Significantly differentially expressed embedded miRNA-mRNA pairs

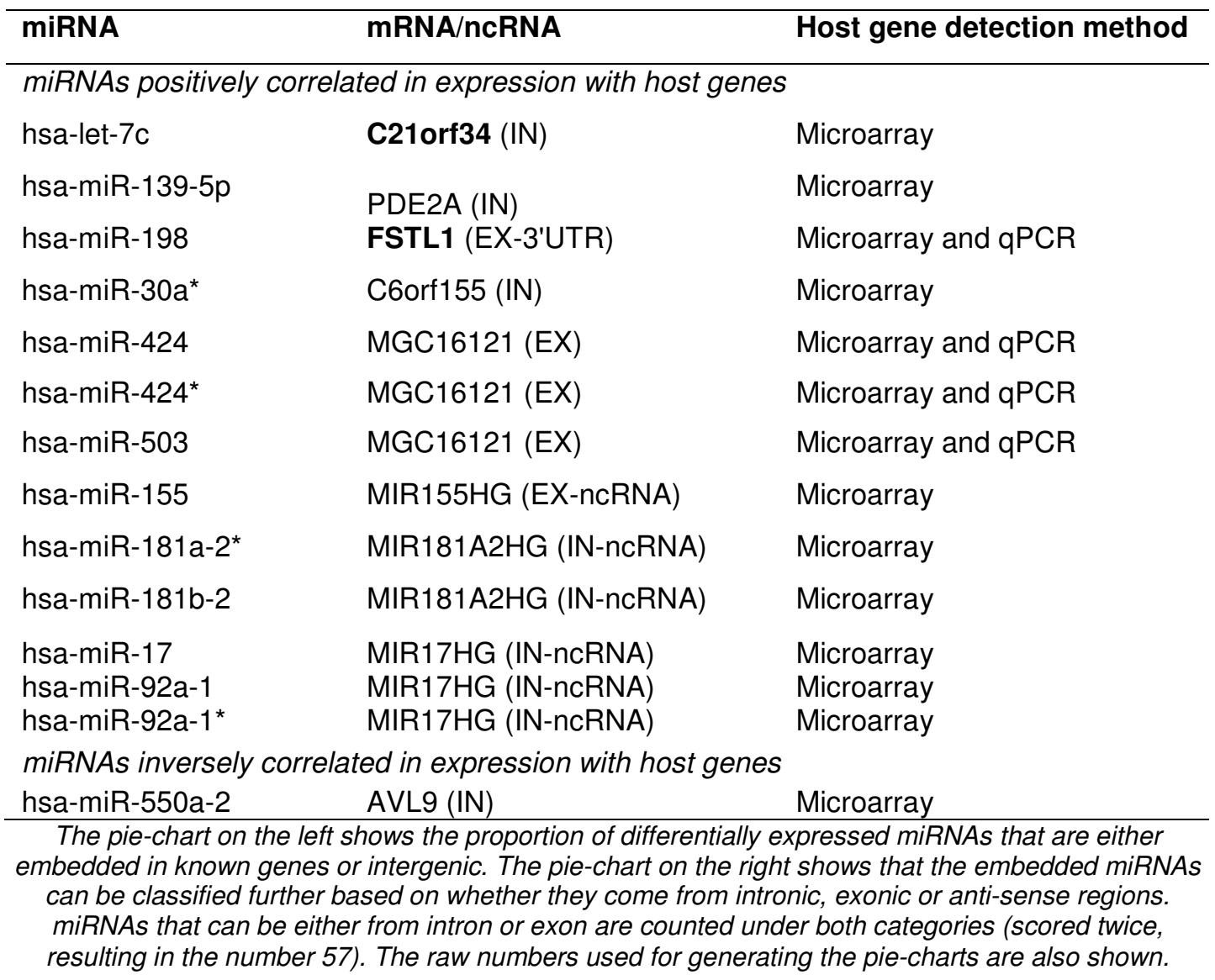




\section{3 miR-7 Regulates Expression of Some miRNAs by Altering the Level of the Transcription Factor RELA/NF-kB}

We found that transfection of miR-7 into HEY cells results in significant changes in the expression levels of 1368 genes including genes known to encode transcription factors previously linked with cancer onset and progression (Table S3; (Karin and Greten, 2005)). Since miRNAs are under transcriptional regulatory control (Krol et al., 2010), transcription factor genes regulated by miR-7 may exert indirect regulatory effects on other miRNAs. To explore this possibility, we selected RELA (v-relreticuloendotheliosis viral oncogene homolog A, aka, Nuclear factor NF-kappa-B p65 subunit) one of the transcription factor coding genes that was significantly differentially expressed after miR-7 transfection in HEY cells (this study) (Table 2) and previously in A549 lung cancer cells ((Webster et al., 2009), see also Table S4). Moreover, RELA is a component of the well-studied transcription factor NF-kB that is a regulator of miRNA expression (Mott et al., 2010; Pacifico et al., 2010; Zhou et al., 2009). Significant down-regulation of RELA in miR-7 transfected HEY cells was confirmed by qPCR and immunoblot assays (Figure 4).

We found that $17(68 \%)$ of the 25 intergenic miRNAs and $38(69 \%)$ of our 55 embedded miRNAs (Figure 5A; Tables S2 and S5) map to within $10 \mathrm{~kb}$ of experimentally validated
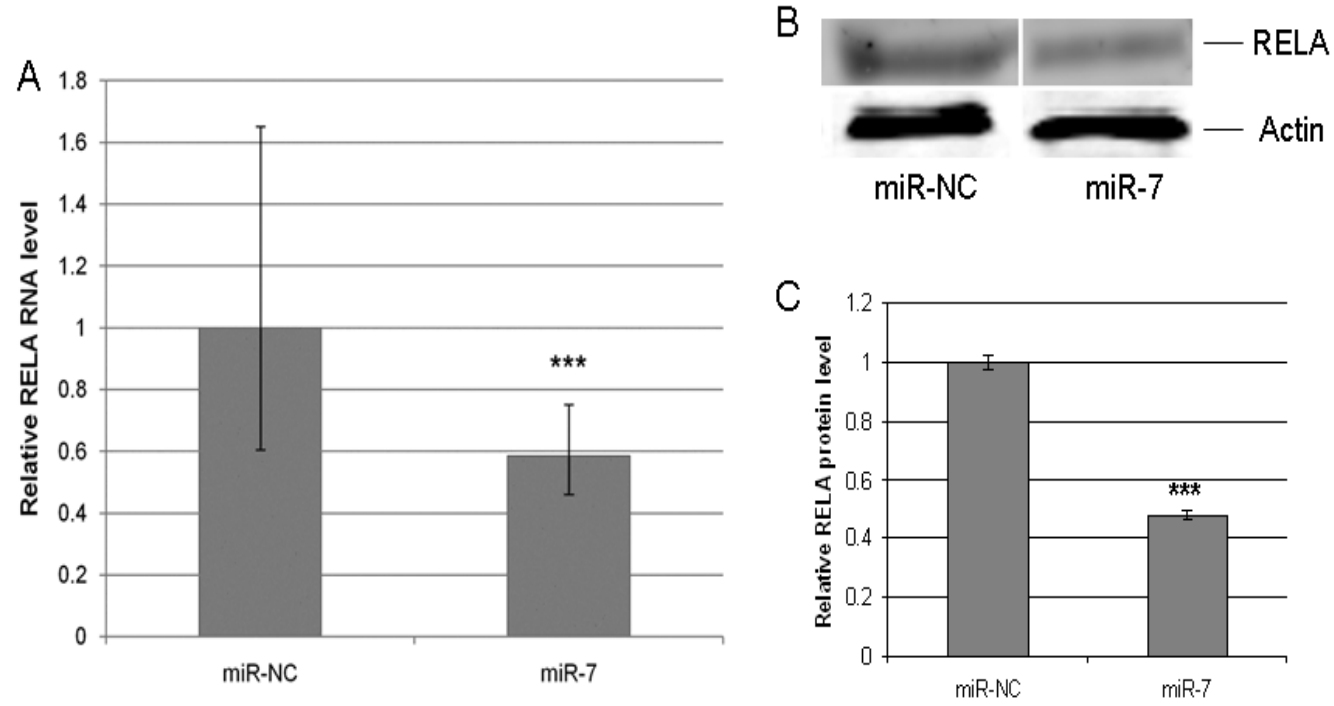

Figure 4. Transfection of miR-7 down-regulates RELA/NF-KB in HEY cells

$R E L A$ expression levels in Hey cells 48 hours after transfection with miR-7 or miR-NC are analyzed by $q P C R(A)$ and Western blot ( $B$ and $C$ ). In both cases RELA expression is down-regulated significantly $\left({ }^{* * *} p<0.001\right)$ after miR-7 transfection compared to negative control (miR-NC) transfection. Bar graphs in $C$ show the relative intensity of blots in B. RELA expression is normalized to GAPDH used as endogenous control (A), or $\beta$-Actin used as loading control (C). Error bars reflect standard deviation of the means calculated from 3 independent replicate samples. 
Table 2. Differentially expressed transcription factors following miR-7 transfection

\begin{tabular}{|c|c|c|c|}
\hline Gene Symbol & miR7-miR-NC & miR7tgts_miRanda & $\begin{array}{l}\text { ENCODE ChIP- } \\
\text { seq Data }\end{array}$ \\
\hline $\mathrm{BACH} 1$ & -0.45255 & & \\
\hline CNOT6 & -0.60309 & $x$ & \\
\hline CNOT8 & -1.26668 & $x$ & \\
\hline CREBL2 & 0.433388 & & \\
\hline IFT57 & 0.664064 & & \\
\hline IRF2BP2 & -0.43606 & & \\
\hline & -0 & & \\
\hline KLF3 & 60824 & & \\
\hline KLF4 & -1.06432 & $x$ & \\
\hline LITAF & -1.52641 & & \\
\hline NOTCH2 & -0.7116 & $x$ & \\
\hline PBX1 & 1.134572 & $x$ & \\
\hline PPM1A & -0.52971 & & \\
\hline RELA & -1.08425 & $x$ & $E$ \\
\hline SETD8 & -1.07335 & $x$ & \\
\hline SP1 & -0.42641 & $x$ & $E$ \\
\hline TAF4B & -0.54876 & & \\
\hline TLE4 & -0.61978 & & \\
\hline UBTF & 0.614296 & & \\
\hline VGLL4 & -0.72098 & & \\
\hline ZBTB20 & 0.399861 & & \\
\hline ZNF512 & -0.49876 & & \\
\hline ZNF580 & 0.457916 & & \\
\hline
\end{tabular}

Listed are the 22 transcription factors identified as being significantly differentially expressed (1.3 fold change, $p<0.05$ ) in both the miR-7 transfected HEY cells (this study) and previously in the A549 lung cancer cells (Webster et al 2009). Differences between the log2 signal values for each gene in HEY cells transfected with either miR-7 or miR-NC is given in the 'miR7-miR-NC'. Of the 22 transcription factors, only 8 have miRanda predicted miR-7 binding sites (marked with ' $x$ ') and only 2, RELA and $S P 1$ have ChIP-seq data showing genome-wide binding sites (marked with ' $E$ ').

(ChIP-seq) RELA/NF-KB binding sites (Birney et al., 2007). To determine if this distribution is greater than expected by chance, we randomly selected 70 human miRNAs from the 718 listed in the UCSC browser and asked how many are located within 10kb of experimentally validated (ChIP-seq) RELA/NF-KB binding sites. After repeating this process 100,000 times, we found that the results displayed a normal distribution with a mean of 43 (Figure S3). The observed number of miRNAs mapping within $10 \mathrm{~kb}$ of experimentally validated (ChIP-seq) RELA/NF-KB binding sites, $55(17+38=55)$, is significantly greater than $43(p<0.003)$ indicating an enrichment of miRNAs within $10 \mathrm{~kb}$ of RELA/NF-KB binding sites (Figure S2).

Nearly half (33 out of 71 ) of the miRNAs that were differentially expressed in our miR-7 transfection experiment have previously been reported to be altered when NF-KB is induced or inhibited in other cellular contexts (Gao et al., 2010; Kluiver et al., 2007; Mott et al., 2010; Pacifico et al., 2010; Wang et al., 2009; Wang et al., 2010; Zhou et al., 2009). Collectively, 
these findings suggest that at least some of the changes in miRNA levels observed after miR-7 transfection into HEY cells are likely mediated by the RELA/NF-kB transcription factor.
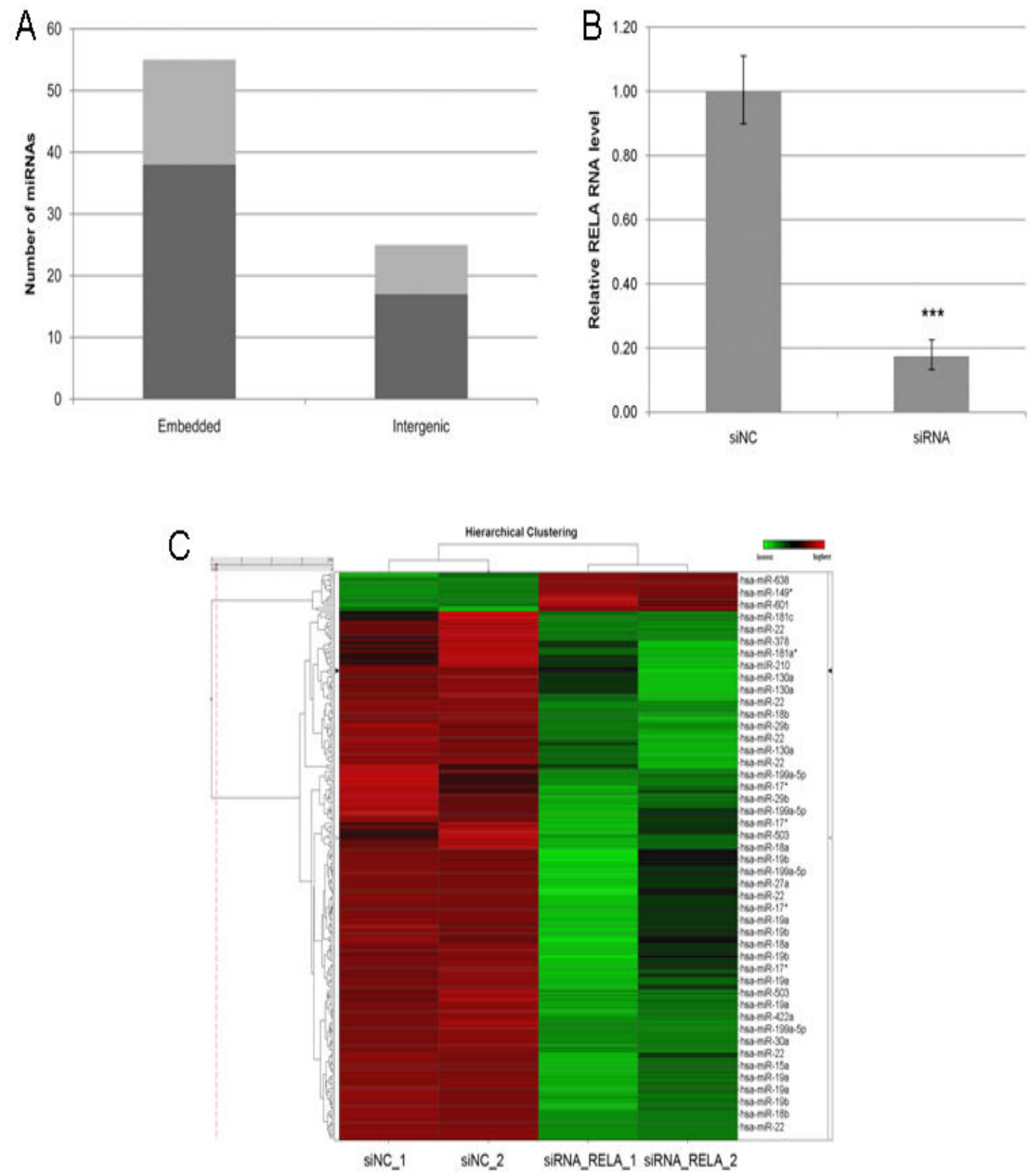

Figure 5.miR-7 regulates expression of some miRNAs by altering the level of RELA. A. Number of embedded and intergenic differentially expressed miRNAs with (dark grey) or without (light grey) NF-KB binding sites within $\pm 10 \mathrm{~kb}$. The number of differentially expressed miRNAs (fold change $\geq 1.4, F D R \leq 5 \%$ ) following miR-7 transfection that are either embedded in coding/non-coding genes or intergenic is represented by the height of the bars. B. qPCR confirmation of successful downregulation of RELA expression 48 hours following transfection of HEY cells with RELA siRNA or SiNC.

C. Significantly differentially expressed (fold change $\geq 1.4$ and FDR $\leq 5 \%$ ) miRNAs in HEY cells following transfection with RELA siRNA or siNC were used for hierarchical clustering of samples. The two samples transfected with siNC are on the left and the two transfected with RELA siRNAare on the right. Some of the IDs of the human miRNAs homologous to the sequences specified by the probesets are shown on the right side of the heat map (See also Table S6). (Note that due to the Affymetrix multiple probe design, some microRNAs are listed more that once) The relative expression of RELA in(B) was measured using GAPDH as endogenous control. Error bars reflect standard deviation of the means calculated from 3 replicate samples. ${ }^{* * *} p<0.001$. 
To further investigate this hypothesis, we transfected HEY cells with RELAsiRNA or a negative control siRNA and collected total RNA (48 hrs post-transfection) for microarray analysis (AffymetrixGeneChip miRNA array). Knock down of RELA expression was confirmed by qPCR (Figure $5 \mathrm{~B}$ ). The results of the microarray analysis demonstrated that 234 miRNA probesets corresponding to 51 known human miRNAs (Figure 5C; Table S6)

Table 3. Significantly differentially expressed miRNAs altered by both miR-7 transfection and knock-down by RELA siRNA

\begin{tabular}{l}
\hline \multicolumn{1}{c}{ Mature miRNAs altered by miR-7 transfection and RELA knock-down } \\
\hline hsa-miR-210 \\
hsa-miR-503 \\
hsa-miR-99b* \\
hsa-miR-181c \\
hsa-miR-146a \\
hsa-miR-193a-3p \\
hsa-miR-199a-5p \\
\hline
\end{tabular}

miRNAs transcribed from the same pre-miRNA

\begin{tabular}{ll} 
RELA knock down & miR-7 transfection \\
\hline hsa-miR-29b(-1) & hsa-miR-29b-1* \\
hsa-miR-140-3p & hsa-miR-140-5p \\
hsa-miR-149* & hsa-miR-149 \\
hsa-miR-17* & hsa-miR-17 \\
hsa-miR-199a-5p & hsa-miR-199a-3p, hsa-miR-199a-5p \\
hsa-miR-30a & hsa-miR-30a* \\
hsa-miR-30e & hsa-miR-30e* \\
\hline
\end{tabular}

miRNAs transcribed from the same cluster

\begin{tabular}{|c|c|c|}
\hline Cluster & RELA knock down & miR-7 transfection \\
\hline miR-17 92 & hsa-miR-17* & hsa-miR-17 \\
\hline miR-17 92 & hsa-miR-18a* & hsa-miR-92a(-1) \\
\hline $\mathrm{miR}-17 \sim 92$ & hsa-miR-18a & hsa-miR-92a- $1^{*}$ \\
\hline miR-17 92 & hsa-miR-19a & \\
\hline miR-17 92 & hsa-miR-19b(-1) & \\
\hline $\mathrm{miR}-18 \sim 106$ & hsa-miR-18b & hsa-miR-20b \\
\hline $\operatorname{miR}-18 \sim 106$ & hsa-miR-19b(-2) & hsa-miR-92a(-2) \\
\hline $\mathrm{miR}-15-16$ & hsa-miR-16(-2) & hsa-miR-15b \\
\hline let-7c, miR-99a & hsa-miR-99a & hsa-let-7c \\
\hline miR-181a-1, miR-181b-1 & hsa-miR-181a* & hsa-miR-181b(-1) \\
\hline miR-130b, miR-301b & hsa-miR-130b & hsa-miR-301b \\
\hline $\mathrm{miR}-424 \sim 542$ & hsa-miR-503 & $\begin{array}{l}\text { hsa-miR-424, hsa-miR- } \\
424^{*} \text {, hsa-miR-503 }\end{array}$ \\
\hline$m i R-181 c, m i R-181 d$ & hsa-miR-181c & $\begin{array}{l}\text { hsa-miR-181c, hsa-miR- } \\
\text { 181d }\end{array}$ \\
\hline \multicolumn{3}{|c|}{$\begin{array}{l}\text { The lists of miRNAs that were significantly differentially expressed (fold change } \geq 1.4, F D R \leq 5 \% \text { ) after } \\
\text { either miR-7 or RELA siRNA transfection were compared and the miRNAs that were altered in both, } \\
\text { had the same pre-miRNA, or that originated in the same cluster are listed. miRNA names followed by } \\
\text { parenthesis containing a number refers to a corresponding paralogous miRNA (for e.g., hsa-miR-19b( } \\
\text { 2) refers to hsa-miR-19b originating from hsa-miR-19-2). }\end{array}$} \\
\hline
\end{tabular}


were significantlydifferentially expressed (fold change $\geq 1.4$, FDR $\leq 5 \%$ ) after $R E L A s i R N A$ transfection relative to controls. Seven of the miRNAs displaying changes in expression after miR-7 transfection displayed similar changes in expression after transfection with RELAsiRNA (Table 3). Twenty additional miRNAs that displayed significant changes in expression after RELAsiRNA transfection either originate from the same pre-miRNA transcript or are clustered within thesame locus $(<10 \mathrm{~kb})$ with 18 miRNAs displaying significant changes in expression after miR-7transfection (Table 3 ). While exogenous expression of miR-7 in HEY cells is expected to modulate the expression a number of genes in addition to RELA (see above), our findings are consistent with the hypothesis that a significant number of the changes in miRNA levels observed after miR-7 transfection into HEY cells are likely mediated by RELA.

\section{4 miR-7 May Regulate Expression of miRNAs by Altering Levels of the Splicing Factor SRSF1 (SF2/ASF)}

Another possible example of a gene regulated by miR-7 that may, in turn, exert indirect regulatory effects on other miRNAs is SRSF1 (serine/arginine-rich splicing factor 1). Wu et al. (2010) recently reported that SRSF1 (SF2/ASF), a gene involved in the splicing of premRNAs (Ge and Manley, 1990; Krainer et al., 1990; Zhou et al., 2002), can form a negative feedback circuit with miR-7. Over-expression of SRSF1 in HeLa cells was shown to result in significant alterations in levels of several (40) miRNAs including a significant elevation in levels of miR-7. Wu et al. (2010) went on to show that elevation in levels of miR-7 results in the down-regulation of SRSF1 (a predicted target of miR-7) indicating a negative feed-back loop.

Since our microarray results (confirmed by qPCR and Western blot analyses, Figure 6) indicate that SRSF1 is significantly down-regulated in miR-7 transfected cells, it is possiblethat at least some of the miR-7 induced changes in levels of miRNAs in these cells may have been mediated by SRSF1. Consistent with this hypothesis, we found that 12 of the 40 miRNAsreported by Wu et al. (2010) to display significant changes in levels of expression in HeLa cells after SRSF1 over-expression also display significant changes in expression after miR-7 transfection in HEY cells (Table 4). In addition, we found that nine additional miRNAs differentially expressed after miR-7 transfection map to primary transcripts containing eightof the differentially expressed miRNAs detected in the Wu et al. (2010) study (Tables 4 and S2).

To further explore the possibility that some of the miR-7 induced changes in miRNA levels in transfected HEY cells may have been mediated by SRSF1, we overlaid the positions of experimentally validated (CLIP-seq or cross-linked immunoprecipitation and sequencing) SRSF1 binding sites (Sanford et al., 2009) with the genomic locations of miRNAs displayingsignificant changes in levels of expression after miR-7 transfection. The results indicate that SRSF1 binding sites are located within the pri-miRNAs of 22 miRNAs (20 embedded, 2 intergenic) displaying significant changes in expression after miR-7 transfection (Table S7). Collectively, these findings are consistent with the hypothesis that some of the miR-7 induced changes in miRNA levels in transfected HEY cells are likely mediated by miR-7 induced changes in levels of SRSF1. 

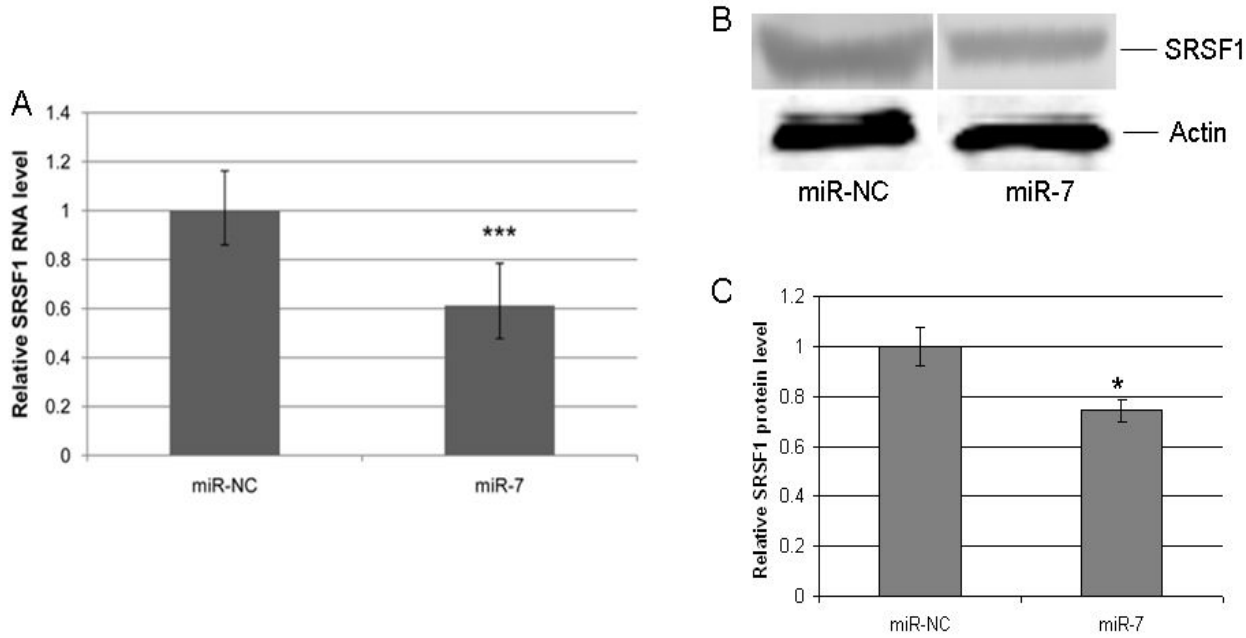

Figure 6. Transfection of miR-7 down-regulates SRSF1 in HEY cells.

SRSF1 expression levels in Hey cells 48 hours after transfection with miR-7 or miR-NC are analyzed by $q P C R(A)$ and Western blot ( $B$ and $C)$. In both cases SRSF1 expression is down-regulated significantly $\left({ }^{*} P<0.05 ;{ }^{* * *} p<0.001\right)$ after miR-7 transfection compared to negative control (miR-NC) transfection. Bar graphs in $C$ show the relative intensity of blots in B. SRSF1 expression is normalized to GAPDH used as endogenous control (A), or $\beta$-Actin used as loading control (C). Error bars reflect standard deviation of the means calculated from 3 independent replicate samples.

Table 4. Significantly differentially expressed miRNAs altered by both miR-7 transfection and SRSF1 induction

miRNAs altered in SRSF1 induction

\section{hsa-let-7c}

hsa-miR-1246

hsa-miR-137

hsa-miR-140-5p

hsa-miR-155

hsa-miR-210

hsa-miR-224

hsa-miR-33a

hsa-miR-551b

hsa-miR-181c

hsa-miR-374b

hsa-miR-92a

hsa-miR-221

hsa-miR-29b

hsa-miR-7

hsa-miR-191

hsa-miR-125a-5p

\section{miRNAs altered in miR-7 transfection}

\author{
hsa-let-7c \\ hsa-miR-1246 \\ hsa-miR-137 \\ hsa-miR-140-5p \\ hsa-miR-155 \\ hsa-miR-210 \\ hsa-miR-224 \\ hsa-miR-33a \\ hsa-miR-551b \\ hsa-miR-181c, hsa-miR-181d \\ hsa-miR-374b, hsa-miR-421 \\ hsa-miR-92a, hsa-miR-92a-1*, hsa-miR-17 \\ hsa-miR-221* \\ hsa-miR-29b-1* \\ hsa-miR-7-1* \\ hsa-miR-425* \\ hsa-miR-99b*
}

The miRNAs that were significantly differentially expressed in either HEY cells after miR-7 transfection (fold change $\geq 1.4$, FDR $\leq 5 \%$ ) (this study) or in HeLa cells (previously reported by Wu et al. (2010) following SRSF1 induction (fold change $\geq 1.5$, FDR $\leq 1 \%$, criterion used in the) were compared and the miRNAs that were altered in both or had the same putative pri-miRNA are listed. Mature miRNAs differentially expressed after both miR-7 transfection and SRSF1 induction are written in bold letters. 


\section{DISCUSSION}

Previously it has been shown that a single miRNA can alter the expression of hundreds of genes (Baek et al., 2008; Lim et al., 2005; Selbach et al., 2008). While some of these changes can be attributed to canonical 3' UTR miRNA targeting, the vast majority ( 70-80\%) of genes altered by a miRNA may be indirectly regulated (Chen et al., 2011) (Shahab et al. 2011). In this study, we employ global gene expression analyses to explore the possibility that one mechanism that may be contributing to these "off-target" effects is miRNA-miRNA regulation.

Our experimental approach was to transfect a well-characterized ovarian cancer cell line (HEY) with a single miRNA and to monitor the effect on the expression levels of other miRNAs and mRNAs by microarray analysis. While miRNA regulatory effects operating exclusively at the level of translation will go undetected in our approach, recent studies indicate that nearly all regulatory changes induced by mammalian miRNAs involve modulations in levels of mRNAs (Guo et al., 2010). Thus, we believe that a majority of miR-7 induced regulatory changes are likely being detected by our approach.

We selected miR-7 for use in our transfection experiments because levels of this miRNA previously have been shown to be significantly changed in ovarian (Shahab et al., 2011; Wyman et al., 2009) and other cancers (Chou et al., 2010; Foekens et al., 2008; Jiang et al., 2010; Kefas et al., 2008; Reddy et al., 2008; Veerla et al., 2009; Webster et al., 2009) relative to normal tissue. Gene expression profiling by microarray revealed that transfection of miR-7 into HEY cells resulted in significant changes in expression levels of 71 miRNAs and 1368 mRNAs.

In principle, miRNAs may regulate expression of other miRNAs either directly or indirectly. For example, it has been proposed that if miRNAs share significant sequence complementarity with other miRNAs, the resulting nucleotide pairing could serve as the basis of direct miRNA-miRNA regulation (Lai et al., 2004). We found that among 71 miRNAs differentially expressed in our study, only 3 displayed even partial (38-62\%, Figure 7) Watson-Crick complementarity to miR-7 indicating that miRNA-miRNA pairing is unlikely to be a significant mechanism used by miR-7 to regulate other miRNAs in our study.

The primary mechanism by which miRNAs regulate expression of mRNA-encoding genes is through translational inhibition and associated mRNA degradation (Djuranovic et al., 2011; Fabian et al., 2010). While this mechanism is presumably not applicable to miRNAs and other untranslated RNAs, there have been reports of miRNAs directly serving as activators (Majid et al., 2010; Place et al., 2008) and repressors (Kim et al., 2008) of transcriptional initiation. It is certainly possible that miRNAs may directly influence the expression of other miRNAs by regulating or co-regulating promoters of miRNA genes and/or protein coding genes in which miRNAs are embedded. However, current evidence suggests that a direct role of miRNAs in promotional control occurs relatively infrequently (Tang et al., 2008; Tang and Zen, 2011). Thus, we believe it is more likely that miRNAs may modulate the expression of other miRNAs indirectly by regulating transcription factors or other regulatory proteins that, in turn, exert direct regulatory control on miRNA expression. 


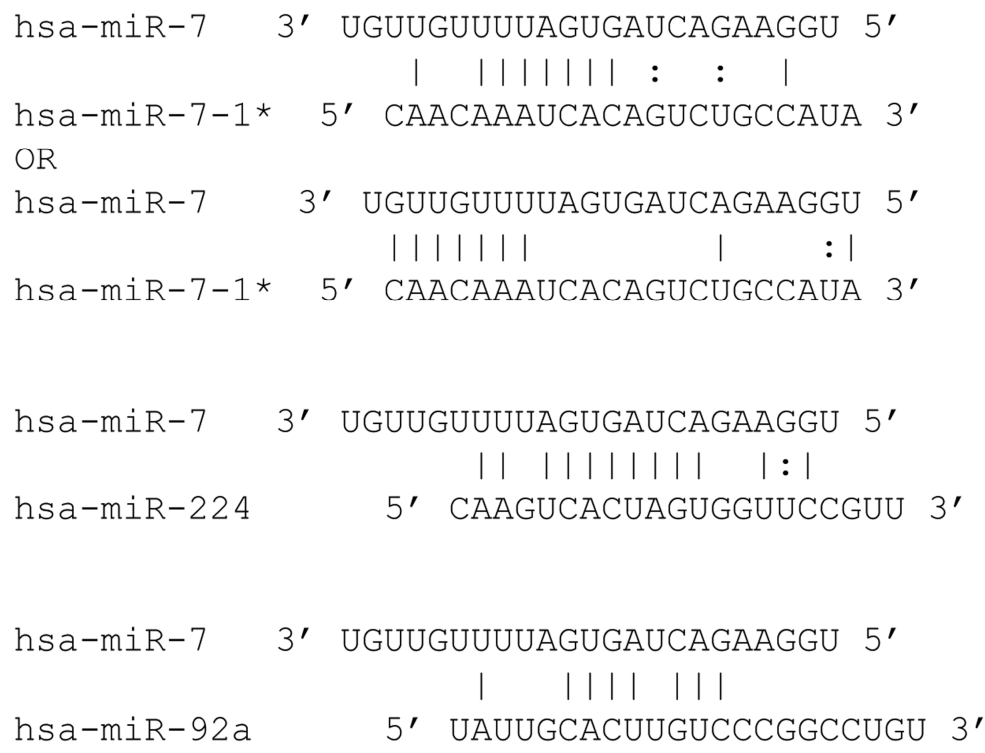

Figure 7. Alignment of three differentially expressed miRNAs with partial complementarity to miR-7.

Sequences of three miRNAs (hsa-miR-7-1*, hsa-miR-224 and hsa-miR-92a) are aligned with miR-7 based on BLAST alignment. Vertical straight lines (I) represents Watson-Crick complementarity and the colon (:) symbol represents GU wobble.

We observed significant changes in expression of 188 transcription factor coding genes after miR-7 transfection. Forty-nine of these, including RELA/NF-KB, are predicted targets of miR7 regulation (Table S3). We selected RELA/NF-KB for further investigation because this transcription factor has been previously implicated in miRNA regulation (Mott et al., 2010; Pacifico et al., 2010; Zhou et al., 2009) and was also found to be down-regulated in a lung cancer cell line following miR-7 transfection (Webster et al., 2009). We found that siRNA knockdown of RELA resulted in a significant change in expression of 51 miRNAs, many of which were also significantly differentially expressed after miR-7 transfection. These results coupled with our finding that $\sim 70 \%$ of the miRNAs differentially expressed after miR-7 transfection map to within $10 \mathrm{~kb}$ of RELA/NF-KB binding sites suggest that this transcription factor plays a significant indirect role in miR-7 induced changes in miRNA expression.

Further support for the hypothesis that miR-7 induced changes in the expression levels of protein coding regulatory genes may play a significant role in affecting changes in levels of miRNAs comes from our analysis of another direct target of miR-7 regulation, the splicing factor SRSF1. We found that SRSF1 binding sites are located within the pri-miRNAs of 25 miRNAs that displayed significant changes in expression after miR-7 transfection into HEY cells. This result coupled with previous findings implicating SRSF1 in the regulation of miR-7 in HeLa cells (Wu et al., 2010) suggests that SRSF1 may also be playing a role in miR-7 induced changes in the expression of miRNAs in HEY cells.

\section{CONCLUSION}

Once considered "junk DNA," a large component of the non-protein coding human genome is now known to encode untranslated RNAs that play important, albeit diverse, roles in gene 
regulation (Eddy, 2001; Mattick, 2007). Among the most intensively studied of these regulatory molecules are short ( 22 nucleotides) regulatory RNAs, called miRNAs. This class of small regulatory RNAs inhibits the translation and/or facilitates the degradation of their targeted messenger RNAs (Fabian et al., 2010). The fact that miRNAs are aberrantly expressed in many cancers has made them attractive candidates for potential use in cancer therapy (Garofalo and Croce, 2011). However, the clinical utility of miRNAs in cancer therapy rests heavily upon our ability to understand and accurately predict the consequences of fluctuations in levels of miRNAs within the context of complex tumor cells. While the regulatory effects of miRNAs in tumor cells is known to be complex (Farazi et al., 2011; Lee and Dutta, 2009; Shahab et al., 2011) the molecular basis of this complexity is only beginning to be explored (Chang et al., 2007; Farazi et al., 2011; Hatley et al., 2010; He et al., 2005; He et al., 2007; Huang et al., 2009).

In this paper, we present evidence from global gene expression analyses that a significant component of this complexity may involve the ability of miRNAs to regulate other miRNAs indirectly through the action of trans-regulatory proteins. Our findings underscore the complexity of miRNA-mediated regulation in cancer cells and the necessity of better understanding the basis of this complexity if we are to fully realize the therapeutic potential of this versatile class of regulatory RNAs.

\section{ACKNOWLEDGEMENTS}

Ovarian Cancer Institute, Ovarian Cycle, The Deborah Nash Harris Endowment Fund, The Robinson Family Foundation, The Waterfall Foundation and The Susan M. Markel Fund provided support for this project. The authors would also like to thank Gordon B. Mills for providing the HEY cells.

\section{COMPETING INTERESTS}

Authors have declared that no competing interests exist.

\section{REFERENCES}

Baek, D., Villen, J., Shin, C., Camargo, F.D., Gygi, S.P., Bartel, D.P. (2008). The impact of microRNAs on protein output.Nature, 455, 64-71.

Bargaje, R., Hariharan, M., Scaria, V., Pillai, B. (2010). Consensus miRNA expression profiles derived from interplatform normalization of microarray data. RNA, 16, 16-25.

Bartel, D.P. (2004). MicroRNAs: genomics, biogenesis, mechanism, and function. Cell, 116, 281-297.

Baskerville, S., Bartel, D.P. (2005). Microarray profiling of microRNAs reveals frequent coexpression with neighboring miRNAs and host genes. RNA, 11, 241-247.

Bergmann, O., Zdunek, S., Alkass, K., Druid, H., Bernard, S., Frisen, J. (2011). Identification of cardiomyocyte nuclei and assessment of ploidy for the analysis of cell turnover.Exp. Cell Res., 317, 188-194.

Betel, D., Wilson, M., Gabow, A., Marks, D.S., Sander, C. (2008). The microRNA.org resource: targets and expression. Nucleic Acids Res., 36, D149-153.

Birney, E., Stamatoyannopoulos, J.A., Dutta, A., Guigo, R., Gingeras, T.R., Margulies, E.H., et al. (2007). Identification and analysis of functional elements in $1 \%$ of the human genome by the ENCODE pilot project. Nature, 447, 799-816. 
Blackburn, W.H., Dickerson, E.B., Smith, M.H., McDonald, J.F., Lyon, L.A. (2009). Peptidefunctionalized nanogels for targeted siRNA delivery. Bioconjug. Chem., 20, 960-968.

Calin, G.A., Croce, C.M. (2006). MicroRNA signatures in human cancers. Nat. Rev. Cancer, $6,857-866$.

Chang, T.C., Wentzel, E.A., Kent, O.A., Ramachandran, K., Mullendore, M., Lee, K.H., et al. (2007). Transactivation of miR-34a by p53 broadly influences gene expression and promotes apoptosis. Mol. Cell, 26, 745-752.

Chen, C., Ridzon, D., Lee, C.T., Blake, J., Sun, Y., Strauss, W.M. (2007). Defining embryonic stem cell identity using differentiation-related microRNAs and their potential targets. Mamm. Genome, 18, 316-327.

Chen, J., Wang, L., Matyunina, L.V., Hill, C.G., McDonald, J.F. (2011). Overexpression of miR-429 induces mesenchymal-to-epithelial transition (MET) in metastatic ovarian cancer cells. Gynecol. Oncol., 121, 200-205.

Chou, Y.T., Lin, H.H., Lien, Y.C., Wang, Y.H., Hong, C.F., Kao, Y.R., et al. (2010). EGFR promotes lung tumorigenesis by activating miR-7 through a Ras/ERK/Myc pathway that targets the Ets2 transcriptional repressor ERF. Cancer Res., 70, 8822-8831.

Colazzo, F., Sarathchandra, P., Smolenski, R.T., Chester, A.H., Tseng, Y.T., Czernuszka, J.T. et al. (2011). Extracellular matrix production by adipose-derived stem cells: implications for heart valve tissue engineering. Biomaterials, 32, 119-127.

Creighton, C.J., Fountain, M.D., Yu, Z., Nagaraja, A.K., Zhu, H., Khan, M., Olokpa, E., Zariff, A., Gunaratne, P.H., Matzuk, M.M., Anderson, M.L. (2011). Molecular profiling uncovers a p53-associated role for microRNA-31 in inhibiting the proliferation of serous ovarian carcinomas and other cancers. Can. Res., 70, 1906-1915.

Dickerson, E.B., Blackburn, W.H., Smith, M.H., Kapa, L.B., Lyon, L.A., McDonald, J.F. (2010). Chemosensitization of cancer cells by siRNA using targeted nanogel delivery. BMC Cancer, 10, 10.

Djuranovic, S., Nahvi, A., Green, R. (2011). A parsimonious model for gene regulation by miRNAs.Science, 331, 550-553.

Eddy, S.R. (2001). Non-coding RNA genes and the modern RNA world. Nat. Rev. Genet., 2, 919-929.

Enright, A.J., John, B., Gaul, U., Tuschl, T., Sander, C., Marks, D.S. (2003).MicroRNA targets in Drosophila.Genome Biol., 5, R1.

Esquela-Kerscher, A., Slack, F.J. (2006).Oncomirs - microRNAs with a role in cancer. Nat. Rev. Cancer, 6, 259-269.

Fabian, M.R., Sonenberg, N., Filipowicz, W. (2010). Regulation of mRNA translation and stability by microRNAs.Annu. Rev. Biochem., 79, 351-379.

Farazi, T.A., Spitzer, J.I., Morozov, P., Tuschl, T. (2011). miRNAs in human cancer. J. Pathol., 223, 102-115.

Foekens, J.A., Sieuwerts, A.M., Smid, M., Look, M.P., de Weerd, V., Boersma, A.W., et al. (2008). Four miRNAs associated with aggressiveness of lymph node-negative, estrogen receptor-positive human breast cancer. Proc. Natl. Acad. Sci. USA, 105, 13021-13026.

Fujita, S., Iba, H. (2008). Putative promoter regions of miRNA genes involved in evolutionarily conserved regulatory systems among vertebrates. Bioinformatics, 24, 303-308.

Gao, C., Zhang, Z., Liu, W., Xiao, S., Gu, W., Lu, H. (2010). Reduced microRNA-218 expression is associated with high nuclear factor kappa B activation in gastric cancer. Cancer, 116, 41-49.

Garofalo, M., Croce, C.M. (2011).microRNAs: Master regulators as potential therapeutics in cancer. Annu. Rev. Pharmacol. Toxicol., 51, 25-43. 
Ge, H., Manley, J.L. (1990). A protein factor, ASF, controls cell-specific alternative splicing of SV40 early pre-mRNA in vitro. Cell, 62, 25-34.

Guo, H., Ingolia, N.T., Weissman, J.S., Bartel, D.P. (2010). Mammalian microRNAs predominately act to decrease target mRNA levels. Nature, 466, 835-840.

Hatley, M.E., Patrick, D.M., Garcia, M.R., Richardson, J.A., Bassel-Duby, R., van Rooij, E. et al. (2010).Modulation of K-Ras-dependent lung tumorigenesis by MicroRNA21.Cancer Cell, 18, 282-293.

He, L., Thomson, J.M., Hemann, M.T., Hernando-Monge, E., Mu, D., Goodson, S., et al, (2005).A microRNA polycistron as a potential human oncogene.Nature, 435, 828-833.

He, L., He, X., Lowe, S.W., Hannon, G.J. (2007). microRNAs join the p53 network--another piece in the tumour-suppression puzzle. Nat. Rev. Cancer, 7, 819-822.

Hevir, N,,Sinkovec, J., Rizner, T.L. (2011). Disturbed expression of phase I and phase II estrogen-metabolizing enzymes in endometrial cancer: lower levels of CYP1B1 and increased expression of S-COMT. Mol. Cell Endocrinol., 331, 158-167.

Huang, T.H., Wu, F., Loeb, G.B., Hsu, R., Heidersbach, A., Brincat, A., et al, (2009). Upregulation of miR-21 by HER2/neu signaling promotes cell invasion. J. Biol. Chem., 284, 18515-18524.

Huang, X., Gschweng, E., Van Handel, B., Cheng, D., Mikkola, H.K., Witte, O.N. (2011). Regulated expression of microRNAs-126/126* inhibits erythropoiesis from human embryonic stem cells. Blood, 117, 2157-2165.

Jiang, L., Liu, X., Chen, Z., Jin, Y., Heidbreder, C.E., Kolokythas, A., et al. (2010). MicroRNA-7 targets IGF1R (insulin-like growth factor 1 receptor) in tongue squamous cell carcinoma cells. Biochem. J., 432, 199-205.

John, B., Enright, A.J., Aravin, A., Tuschl, T., Sander, C., Marks, D.S. (2004). Human MicroRNA targets. PLoS Biol., 2, e363.

Karin, M., Greten, F.R. (2005). NF-kappaB: linking inflammation and immunity to cancer development and progression. Nat. Rev. Immunol., 5, 749-759.

Kefas, B., Godlewski, J., Comeau, L., Li, Y., Abounader, R., Hawkinson, M., et al. (2008).microRNA-7 inhibits the epidermal growth factor receptor and the Akt pathway and is down-regulated in glioblastoma. Cancer Res., 68, 3566-3572.

Kim, D.H., Saetrom, P., Snove, O., Jr., Rossi, J.J. (2008).MicroRNA-directed transcriptional gene silencing in mammalian cells. Proc. Natl. Acad. Sci. USA, 105, 16230-16235.

Kim, V.N., Nam, J.W. (2006). Genomics of microRNA. Trends Genet., 22, 165-173.

Kluiver, J., van den Berg, A., de Jong, D., Blokzijl, T., Harms, G., Bouwman, E., et al. (2007).Regulation of pri-microRNA BIC transcription and processing in Burkitt lymphoma.Oncogene, 26, 3769-3776.

Koppelstaetter, C., Jennings, P., Hochegger, K., Perco, P., Ischia, R., Karkoszka, H., et al, (2005). Effect of tissue fixatives on telomere length determination by quantitative PCR.Mech. Ageing Dev., 126, 1331-1333.

Krainer, A.R., Conway, G.C., Kozak, D. (1990). Purification and characterization of premRNA splicing factor SF2 from HeLa cells. Genes Dev., 4, 1158-1171.

Krol, J., Loedige, I., Filipowicz, W. (2010). The widespread regulation of microRNA biogenesis, function and decay. Nat. Rev. Genet., 11, 597-610.

Lai , E.C., Wiel, C., Rubin, G.M. (2004). Complementary miRNA pairs suggest a regulatory role for miRNA:miRNA duplexes. RNA, 10, 171-175.

Lee, Y.S., Dutta, A. (2009). MicroRNAs in cancer.Annu. Rev. Pathol., 4, $199-227$.

Liang, Y., Ridzon, D., Wong, L., Chen, C. (2007). Characterization of microRNA expression profiles in normal human tissues. BMC Genomics, 8, 166.

Lim, L.P., Lau, N.C., Garrett-Engele, P., Grimson, A., Schelter, J.M., Castle, J., et al. (2005). Microarray analysis shows that some microRNAs downregulate large numbers of target mRNAs. Nature, 433, 769-773. 
Lossos, I.S., Czerwinski, D.K., Alizadeh, A.A., Wechser, M.A., Tibshirani, R., Botstein, D., et al. (2004). Prediction of survival in diffuse large-B-cell lymphoma based on the expression of six genes. N. Engl. J. Med., 350, 1828-1837.

Majid, S., Dar, A.A., Saini, S., Yamamura, S., Hirata, H., Tanaka, Y., et al. (2010). MicroRNA-205-directed transcriptional activation of tumor suppressor genes in prostate cancer.Cancer, 116, 5637-5649.

Mattick, J.S. (2007). A new paradigm for developmental biology. J. Exp. Biol., 210, 15261547.

Micallef, J., Taccone, M., Mukherjee, J., Croul, S., Busby, J., Moran, M.F., et al. (2009). Epidermal growth factor receptor variant III-induced glioma invasion is mediated through myristoylated alanine-rich protein kinase $\mathrm{C}$ substrate overexpression. Cancer Res., 69, 7548-7556.

Mieczkowski, J., Tyburczy, M.E., Dabrowski, M., Pokarowski, P. (2010). Probe set filtering increases correlation between AffymetrixGeneChip and qRT-PCR expression measurements. BMC Bioinformatics, 11, 104.

Monteys, A.M., Spengler, R.M., Wan, J., Tecedor, L., Lennox, K.A., Xing, Y., et al. (2010).Structure and activity of putative intronic miRNA promoters.RNA, 16, 495-505.

Mott, J.L., Kurita, S., Cazanave, S.C., Bronk, S.F., Werneburg, N.W., Fernandez-Zapico, M.E. (2010).Transcriptional suppression of mir-29b-1/mir-29a promoter by c-Myc, hedgehog, and NF-kappaB. J. Cell Biochem., 110, 1155-1164.

Ozsolak, F., Poling, L.L., Wang, Z., Liu, H., Liu, X.S., Roeder, R.G., et al. (2008). Chromatin structure analyses identify miRNA promoters. Genes Dev., 22, 3172-3183.

Pacifico, F., Crescenzi, E., Mellone, S., lannetti, A., Porrino, N., Liguoro, D., et al, (2010). Nuclear factor-\{kappa\}B contributes to anaplastic thyroid carcinomas through upregulation of miR-146a. J. Clin. Endocrinol. Metab., 95, 1421-1430.

Pfaffl, M.W., Horgan, G.W., Dempfle, L. (2002). Relative expression software tool (REST) for group-wise comparison and statistical analysis of relative expression results in realtime PCR. Nucleic Acids Res., 30, e36.

Place, R.F., Li, L.C., Pookot, D., Noonan, E.J., Dahiya, R. (2008). MicroRNA-373 induces expression of genes with complementary promoter sequences. Proc. Natl. Acad. Sci. USA, 105, 1608-1613.

Reddy, S.D., Ohshiro, K., Rayala, S.K., Kumar, R. (2008). MicroRNA-7, a homeobox D10 target, inhibits p21-activated kinase 1 and regulates its functions. Cancer Res., 68, 8195-8200.

Rodriguez, A., Griffiths-Jones, S., Ashurst, J.L., Bradley, A. (2004). Identification of mammalian microRNA host genes and transcription units.Genome Res., 14, 19021910.

Saini, H.K., Griffiths-Jones, S., Enright, A.J. (2007). Genomic analysis of human microRNA transcripts. Proc. Natl. Acad. Sci. USA, 104, 17719-17724.

Sanford, J.R., Wang, X., Mort, M., Vanduyn, N., Cooper, D.N., Mooney, S.D., et al. (2009). Splicing factor SFRS1 recognizes a functionally diverse landscape of RNA transcripts. Genome Res., 19, 381-394.

Saydam, O., Senol, O., Wurdinger, T., Mizrak, A., Ozdener, G.B., Stemmer-Rachamimov, A.O., et al. (2011).miRNA-7 Attenuation in Schwannoma tumors stimulates growth by upregulating three oncogenic signaling pathways. Cancer Res., 71, 852-861.

Selbach, M., Schwanhausser, B., Thierfelder, N., Fang, Z., Khanin, R., Rajewsky, N. (2008). Widespread changes in protein synthesis induced by microRNAs. Nature, 455, 58-63.

Shahab, S.W., Matyunina, L.V., Mezencev, R., Walker, L.D., Bowen, N.J., Benigno, B.B., McDonald, J.F. (2011). Evidence for the complexity of micro-RNA-mediated regulation in ovarian cancer: a systems approach. PLoS One, 6, e22508. 
Shibata, M., Nakao, H., Kiyonari, H., Abe, T., Aizawa, S. (2011). MicroRNA-9 regulates neurogenesis in mouse telencephalon by targeting multiple transcription factors. J. Neurosci., 31, 3407-3422.

Tang, G., Tang, X., Mendu, V., Jia, X., Chen, Q.J., He, L. (2008). The art of microRNA: various strategies leading to gene silencing via an ancient pathway. Biochim. Biophys. Acta, 1779, 655-662.

Tang, R., Zen, K. (2011). Gold glitters everywhere: nucleus microRNAs and their functions. Frontiers in Biology, 6, 69-75.

Tavazoie, S.F., Alarcon, C., Oskarsson, T., Padua, D., Wang, Q., Bos, P.D., et al. (2008). Endogenous human microRNAs that suppress breast cancer metastasis. Nature, 451, 147-152.

Veerla, S., Lindgren, D., Kvist, A., Frigyesi, A., Staaf, J., Persson, H., et al. (2009). MiRNA expression in urothelial carcinomas: important roles of miR-10a, miR-222, miR-125b, miR-7 and miR-452 for tumor stage and metastasis, and frequent homozygous losses of miR-31. Int. J. Cancer, 124, 2236-2242.

Visone, R., Croce, C.M. (2009). MiRNAs and cancer. Am. J. Pathol., 174, 1131-1138.

Wahid, F., Shehzad, A., Khan, T., Kim, Y.Y. (2010). MicroRNAs: synthesis, mechanism, function, and recent clinical trials. Biochim. Biophys. Acta, 1803, 1231-1243.

Wang, B., Majumder, S., Nuovo, G., Kutay, H., Volinia, S., Patel, T., et al. (2009). Role of microRNA-155 at early stages of hepatocarcinogenesis induced by choline-deficient and amino acid-defined diet in C57BL/6 mice. Hepatology, 50, 1152-1161.

Wang, B.D., Kline, C.L., Pastor, D.M., Olson, T.L., Frank, B., Luu, T., et al. (2010). Prostate apoptosis response protein 4 sensitizes human colon cancer cells to chemotherapeutic 5-FU through mediation of an NF kappaB and microRNA network. Mol. Cancer, 9, 98.

Webster, R.J., Giles, K.M., Price, K.J., Zhang, P.M., Mattick, J.S., Leedman, P.J. (2009). Regulation of epidermal growth factor receptor signaling in human cancer cells by microRNA-7. J. Biol. Chem., 284, 5731-5741.

Wiemer, E.A. (2007). The role of microRNAs in cancer: no small matter. Eur. J. Cancer, 43, 1529-1544.

Wilson, J.A., Zhang, C., Huys, A., Richardson, C.D. (2011). Human Ago2 is required for efficient microRNA 122 regulation of hepatitis $C$ virus RNA accumulation and translation. J. Virol., 85, 2342-2350.

Wu, H., Sun, S., Tu, K., Gao, Y., Xie, B., Krainer, A.R., et al. (2010).A splicing-independent function of SF2/ASF in microRNA processing. Mol. Cell, 38, 67-77.

Wyman, S.K., Parkin, R.K., Mitchell, P.S., Fritz, B.R., O'Briant, K., Godwin, A.K., et al. (2009). Repertoire of microRNAs in epithelial ovarian cancer as determined by next generation sequencing of small RNA cDNA libraries. PLoS ONE, 4, e5311.

Zhou, R., Hu, G., Liu, J., Gong, A.Y., Drescher, K.M., Chen, X.M. (2009). NF-kappaB p65dependent transactivation of miRNA genes following Cryptosporidium parvum infection stimulates epithelial cell immune responses. PLoSPathog., 5, e1000681.

Zhou, Z., Licklider, L.J., Gygi, S.P., Reed, R. (2002). Comprehensive proteomic analysis of the human spliceosome.Nature, 419, 182-185. 


\section{APPENDIX}

SUPPLEMENTARY INFORMATION IS AVAILABLE AT THE WEBSITE OF BRITISH JOURNAL OF MEDICINE AND MEDICAL RESEARCH.

(C) 2012 Shahab et al.; This is an Open Access article distributed under the terms of the Creative Commons Attribution License (http://creativecommons.org/licenses/by/3.0), which permits unrestricted use, distribution, and reproduction in any medium, provided the original work is properly cited. 